\title{
Innovation in CryoEM Sample Preparation: Towards Commercialization of Spotiton
}

Russell S. King ${ }^{1}$, John P. Moore ${ }^{1}$, Klaus Doering${ }^{1}$, R. John Walker ${ }^{1}$, Michele C. Darrow ${ }^{1}$, Melanie A. Adams-Cioaba ${ }^{1}$

1. TTP Labtech LTD., Melbourn Science Park, Hertfordshire, UK

Protein structure determination is fundamental to the understanding of function, providing context for both individual proteins and within large biologically relevant complexes. Structure determination is, thus, critical both for target discovery and validation along with guiding the development of new small molecule and biologic therapeutics.

While X-ray crystallography continues to be a standard resource for high resolution structure determination, cryo-electron microscopy (cryoEM) has matured in recent years to become a viable alternative for targets of broad molecular mass and at ever-increasing resolution. Recent developments in the field have automated and streamlined the data collection and analysis processes, while direct electron detectors and their associated improvements in resolution have now become standard practice.

Despite these advances in both microscopes and computational approaches, sample preparation remains highly challenging and a barrier to broader adoption of the method beyond traditional cryoEM laboratories. To meet this goal, ease-of-use improvements, such as reducing manual grid handling and variability between grid freezing sessions, are necessary. Sample preparation approaches that may lessen the challenges of preferential orientation due to interactions with either the support film or the air-water interface will be essential to enabling consistent structure determination for broad ranges of targets.

The development of a blotting-free approach to cryoEM sample preparation has been previously described [1-2]. The instrument employs a novel approached for vitrifying samples for cryoEM in which picolitre amounts of samples are applied to self-blotting grids ${ }^{3}$, allowing for rapid vitrification, minimal sample handling and removing the need for buffer-removal via blotting or evaporation prior to freezing.

We provide here a development update on both the Spotiton instrument and the associated self-blotting grids. We highlight recent data from collaborating groups on the benefits of the approach using challenging samples from a variety of target classes. We concentrate on recent platform updates that capture sample behavior on the cryoEM grid and that may lead to sample application tuning that is predictive of downstream ice quality post-vitrification.

\section{References:}

[1] T Jain et al, J Struct Bio 179 (2012), p. 68

[2] I Razinkov et al, J Struct Bio 195 (2016), p. 190 


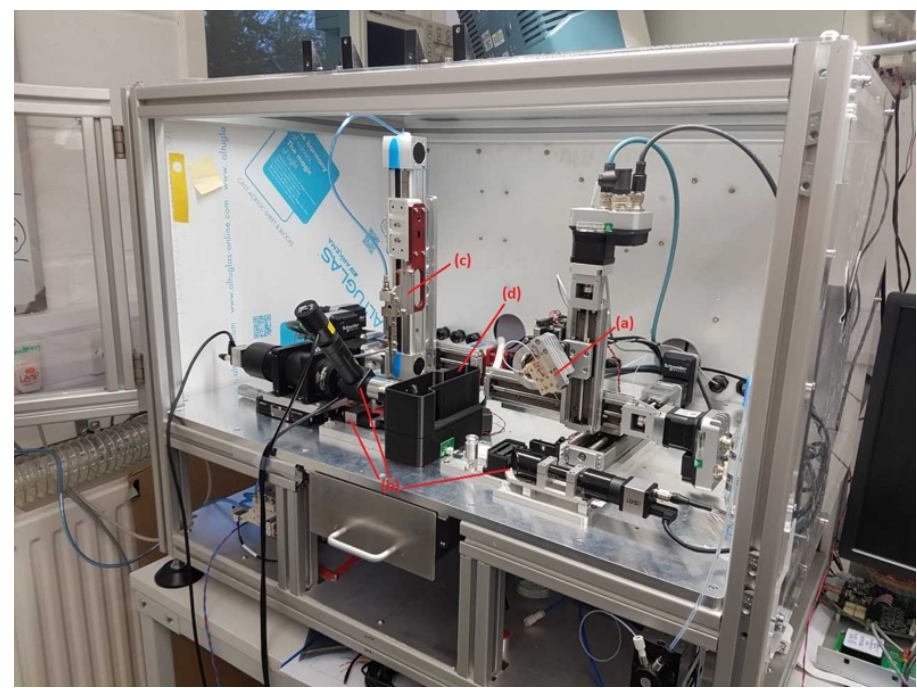

Figure 1. An early version of the Spotiton system showing the (a) picoliter dispensing unit, (b) image capture systems, (c) high speed plunge robot (d) localized environmental control

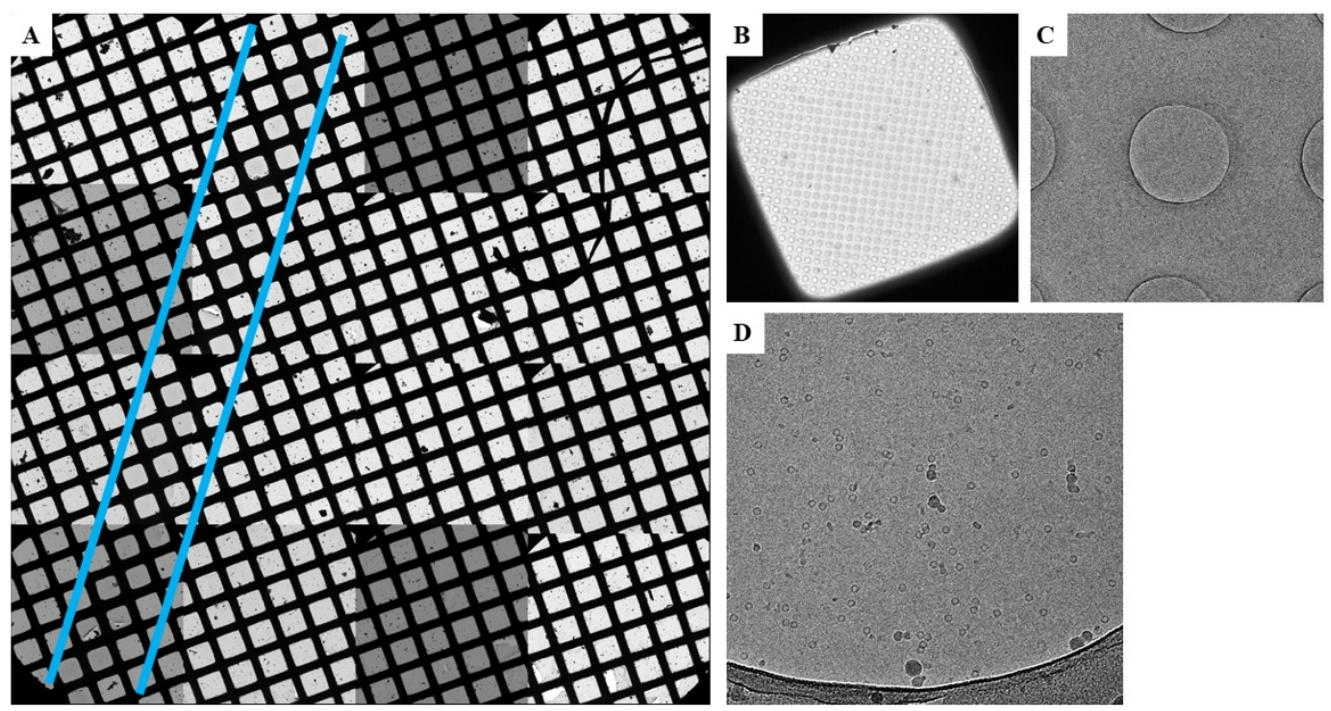

Figure 2. Example apoferritin sample grid vitrified using a Spotiton prototype. (a) Low magnification grid mosaic with sample stripe marked by blue lines. (b) Representative image of a grid square with approximately 500 useable holes for high-resolution data collection. (c) Representative image of well vitrified grid holes. (d) Representative image of apoferritin vitrified by the Spotiton system using selfblotting grids. 